\title{
¿Neumobilia o gas en sistema venoso portal? Implicaciones clínicas, diagnósticas y terapéuticas
}

\section{Pneumobilia or portal venous gas? Clinical, diagnostic and therapeutic implications}

\author{
Denzil Garteiz Martínez, * Óscar Alejandro Mora Torres, ${ }^{\ddagger}$ \\ Alejandro Weber Sánchez, ${ }^{\S}$ Mario Calva Arcos, ${ }^{\Uparrow}$ Alejandro Zatarain Gulmar"
}

\begin{abstract}
Citar como: Garteiz MD, Mora TÓA, Weber SA, Calva AM, Zatarain GA. ¿Neumobilia o gas en sistema venoso portal? Implicaciones clínicas, diagnósticas y terapéuticas. Acta Med GA. 2022; 20 (1): 74-78. https://dx.doi.org/10.35366/103561
\end{abstract}

\section{Resumen}

Introducción: La diferenciación, en la interpretación radiológica, entre la neumobilia y el gas portal tiene implicaciones clínicas y terapéuticas de gran importancia. El presente trabajo tiene por objetivo describir los criterios radiológicos y clínicos más relevantes de estas dos entidades, con el fin de disminuir la confusión que puede existir entre ellas. Caso clínico: Paciente masculino de 40 años con cuadro gastroenteral en cuya radiografía simple de abdomen se malinterpretan unas zonas radiolúcidas del parénquima hepático como neumobilia y se provoca un aumento innecesario de estudios y comorbilidades. Conclusión: Diferenciar entre neumobilia y gas portal es un reto que exige la cooperación estrecha entre radiólogos y clínicos. Ambas circunstancias pueden estar relacionadas con patologías de un amplio espectro de severidades y, por lo tanto, la integración de un diagnóstico debe ser cuidadosamente complementado entre la clínica y los resultados de imagen.

Palabras clave: Neumobilia, gas portal, radiología, diagnóstico.

\section{INTRODUCCIÓN}

La diferenciación en la interpretación radiológica entre la neumobilia y el gas portal tiene implicaciones clínicas y terapéuticas de gran importancia. Una confusión en el diagnóstico puede llevar al tratamiento excesivo de

\section{Abstract}

Introduction: The differentiation, in the radiological interpretation, between pneumobilia and portal gas has clinical and therapeutic implications of great importance. The present work is focused on describing the most relevant radiologic and clinical criteria of these two entities, with the objective of reducing confusion among them. Case report: A 40-year-old male patient, with gastrointestinal symptoms, whose simple abdominal X-ray is misinterpreted as having pneumobilia due to the presence of radiolucent areas in the liver parenchyma, leading to further unnecessary studies and increased morbidity. Conclusion: Being able to differentiate pneumobilia from portal gas y a challenge that requires close cooperation between clinicians and radiologists. Both circumstances can be related to pathologies with a wide range of severities and therefore, the integration of a diagnosis must be carefully complemented by the clinical scenario and the radiologic images.

Keywords: Pneumobilia, portal venous gas, radiology, diagnosis.

un paciente o a menospreciar un caso que sí presenta gravedad. En este artículo se ilustran los riesgos que pueden surgir a partir de esta malinterpretación y se revisan los criterios descritos en la literatura para poder hacer frente a estos escenarios complejos y poco frecuentes.
* Cirujano General, MSc, FACS. División de Cirugía.

₹ Médico Residente de Cirugía General. Universidad Nacional Autónoma de México.

$\S$ Cirujano General, PhD. División de Cirugía.

" Radiólogo. Departamento de Radiología e Imagen.

\| Médico Residente de Radiología e Imagen. Universidad Nacional Aceptado: 11-02-2021. Autónoma de México.

Hospital Angeles Lomas. Estado de México, México.
Correspondencia:

Dr. Óscar Alejandro Mora Torres

Correo electrónico: alejandromora118@gmail.com

www.medigraphic.com/actamedica

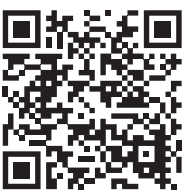




\section{PRESENTACIÓN DE CASO CLÍNICO}

Paciente masculino de 40 años sin antecedentes de importancia que presentó un cuadro de inicio súbito con evacuaciones líquidas, dolor abdominal tipo cólico y malestar general. Se le encontró con signos vitales normales, deshidratación moderada y sin datos de irritación peritoneal o sepsis. Los laboratorios excluyeron leucocitosis, bandemia o alteraciones en las pruebas de función hepática. La radiografía simple de abdomen mostró dilatación de asas de intestino delgado y colon, así como niveles hidroaéreos dispersos, compatibles con íleo sin evidencia de obstrucción intestinal mecánica. El reporte radiológico describió "neumobilia", debido a la presencia de zonas radiolúcidas en el parénquima hepático (Figura 1). Esto motivó la decisión de realizar una tomografía computarizada, la cual se comenta como positiva para "neumobilia" (Figura 2) y, además, se reporta como posible causa la presencia de una "fístula colecistoduodenal", sin que existieran otros hallazgos que sustentaran estos diagnósticos. Sin una indicación clara, se decidió realizar una colangiopancreatografía retrógrada endoscópica (CPRE) que fue reportada como normal, sin alteraciones en la vía biliar. A las 24 horas, el paciente presentó dolor abdominal intenso, elevación de enzimas pancreáticas (amilasa 1,421 UI y lipasa 2,306 UI) y mal estado general. Se diagnosticó pancreatitis aguda leve según los criterios de Atlanta (APACHE II) y grado C según los de Balthazar. El paciente fue trasladado a otro hospital en donde se dio manejo médico a la pancreatitis y fue dado de alta siete días después, encontrándose en buen estado de salud hasta la fecha.

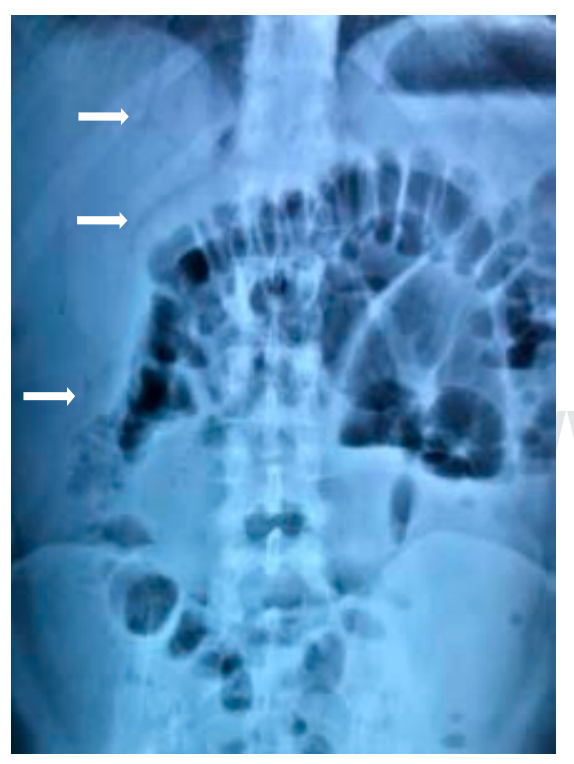

Figura 1:

Radiografía simple de abdomen que muestra aire en sistema venoso portal interpretada inicialmente como neumobilia, se observan zonas radiolúcidas en el parénquima hepático de aparente distribución biliar.

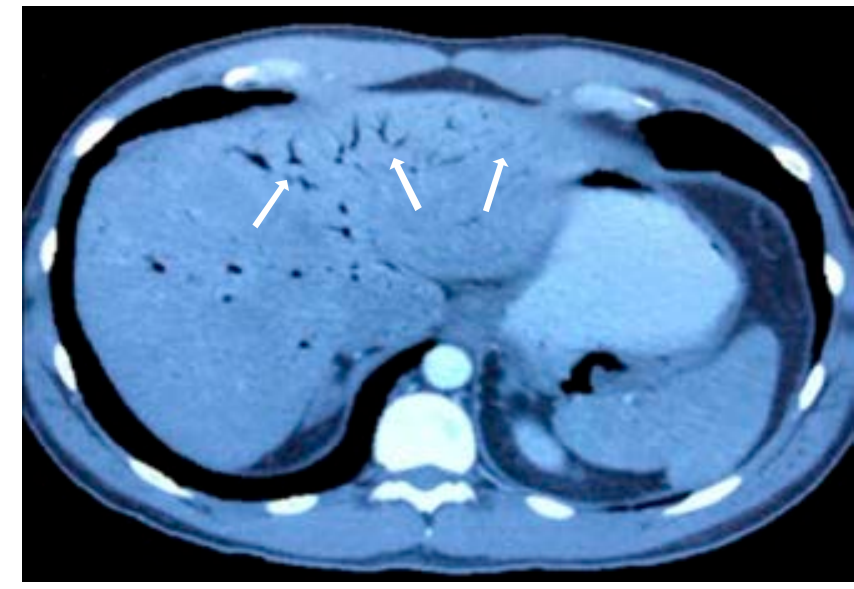

Figura 2: Tomografía computarizada de abdomen en la que se observa un patrón aéreo hacia la periferia del parénquima hepático (interpretada inicialmente con neumobilia), haciéndola sugestiva de gas venoso portal.

\section{DISCUSIÓN}

El caso clínico que presentamos ilustra dos problemas con los cuales los radiólogos y clínicos se pueden enfrentar: la inadecuada diferenciación radiológica entre neumobilia y gas portal, y el sobretratamiento de un paciente basado en un diagnóstico equivocado. En un análisis retrospectivo de los estudios radiológicos, se puede observar que las imágenes radiolúcidas son de predominio periférico (Figura 1) y que existe gas en la circulación venosa perigástrica (Figuras 2 y 3) y portal, mismos que se debieron haber atribuido al cuadro gastroenteral que presentaba el paciente. Esto hubiera evitado la pancreatitis que fue secundaria a una CPRE equivocadamente indicada. Así como en este caso, en la literatura existen ejemplos de abordajes terapéuticos erróneos debido a la confusión diagnóstica inicial. Es por eso que en esta discusión analizaremos los aspectos más relevantes sobre la diferencia clínica, diagnóstica y terapéutica entre neumobilia y gas portal.

Aspectos diferenciales del diagnóstico clínico. El término "neumobilia" se utiliza para describir aire dentro de los conductos biliares, intra- o extrahepáticos, así como en la vesícula biliar. ${ }^{1}$ Por lo general, este aire proviene de alteraciones anatómicas en el esfínter de Oddi, dilatación intestinal o de la comunicación anormal entre una parte del tubo digestivo (duodeno o colon, principalmente) y el árbol biliar. Puede ocurrir de manera iatrógena por instrumentación, lesiones biliares o espontánea, posterior a un síndrome de Mirizzi o al íleo biliar. También puede observarse en pacientes con diarrea biliar crónica asociada a malabsorción y posterior a trauma abdominal contuso. 
En la Tabla 1 se agrupan algunos de los diagnósticos diferenciales asociados a neumobilia.

Por otra parte, la presencia de gas portal se trata de aire en la circulación venosa del sistema porta. Es más frecuente en la infancia (por enterocolitis necrosante) y en el adulto mayor (por isquemia intestinal). El lóbulo izquierdo del hígado es más propenso a contener gas portal debido a la anatomía de la circulación venosa. Su presencia en el sistema venoso portal obedece a tres causas principales: 1) al aumento de la presión intraluminal; 2) intravasación de gas a través de la mucosa dañada; y 3) a translocación bacteriana. ${ }^{2}$ El espectro de causas de gas portal puede ir desde condiciones benignas hasta condiciones que ponen



Figura 3: Gas en la circulación venosa perigástrica, sugestivo de gas en sistema venoso portal. en peligro la vida. En $75 \%$ de los casos es un signo de gravedad, con una mortalidad de $70 \%^{3,4}$ por su asociación con neumatosis o necrosis intestinal con una supervivencia global de $25 \%$ en estas circunstancias. ${ }^{5}$ En el caso del origen intraluminal se ha demostrado que se debe a la presencia de lesiones en la mucosa intestinal asociado con aumento de la presión intraluminal. ${ }^{6}$ Se ha documentado gas portal posterior a procedimientos como el colon por enema y la colonoscopia. Desde la primera descripción de bacterias formadoras de gas en la circulación portal en $1978,{ }^{7}$ se ha señalado que en los casos en que no se presenta necrosis de la pared intestinal, el origen del gas portal pudiera deberse a la presencia de flebitis séptica, abscesos cercanos a la vasculatura portal y metástasis hepáticas infectadas, ${ }^{8}$ asociado además a cuadros agudos gastroenterales con diarrea y vómito severos, en los cuales se ha demostrado su resolución espontánea variable desde minutos o hasta varias semanas. ${ }^{9,10}$ Es claro que las patologías que ocasionan presencia de gas portal tienen, en general, peor pronóstico que las que ocasionan neumobilia (Tabla 2). ${ }^{11}$

Aspectos diferenciales del diagnóstico radiológico. Existen ciertos patrones radiológicos que pueden ayudar a establecer el diagnóstico diferencial entre neumobilia y gas portal; en la radiografía simple de abdomen la neumobilia se manifiesta con la presencia de imágenes radiolúcidas, dentro del parénquima hepático, con un patrón de distribución que semeja al árbol biliar, como ejemplo tenemos el "signo del sable", el cual es una zona radiolúcida ubicada a la derecha de la columna vertebral, a nivel del lóbulo izquierdo del hígado (gas en el conducto hepático izquierdo). ${ }^{12}$ En una tomografía el aire tiende a ser más central y estar a más de $2 \mathrm{~cm}$ de la cápsula hepática

Tabla 1: Condiciones clínicas asociadas a neumobilia.

Con comunicación anatómica anormal

Derivación biliodigestiva

- Colédoco-duodeno anastomosis

- Hepático-yeyuno anastomosis

Fístula bilioentérica

- Colecistoduodenal

- Colecistocolónica

Íleo biliar

Síndrome de Bouveret (obstrucción gástrica por lito proveniente de fístula colédoco-duodenal)

\section{Con comunicación anatómica normal}

Incompetencia del esfínter de Oddi Colecistitis enfisematosa

Colangitis piógena

Absceso hepático

Metástasis hepática infectada

Trauma contuso abdominal

Obstrucción intestinal

Úlcera péptica

Enfermedad de Caisson (enfermedad

por descompresión)

Pielonefritis

Anemia de células falciformes

Linfoma duodenal

Reanimación cardiopulmonar

\section{Secundaria a instrumentación clínica}

Colangiopancreatografía retrógrada endoscópica

Esfinterotomía endoscópica

Colocación de endoprótesis biliares

Endoscopia gastroduodenal 
Tabla 2: Condiciones que pueden asociarse a la presencia de gas venoso portal.

Enfermedades con mayor riesgo de mortalidad
Enfermedades con menor riesgo de mortalidad
Condiciones asociadas a instrumentación clínica
Isquemia intestinal

Enterocolitis necrosante

Pancreatitis aguda

Colangitis supurativa

Diverticulitis complicada

Pileflebitis

Fístula colovenosa

Trasplante hepático

Enfermedad de injerto contra

huésped
Enfermedad de Crohn

Colitis ulcerativa

Fibrosis quística

Epilepsia

Toxicidad por colchicina

Dilatación gástrica aguda

Obstrucción intestinal

íleo paralítico

Absceso intraabdominal

Diarrea
Colangiopancreatografía retrógrada endoscópica

Cirugía laparoscópica

Colocación de endoprótesis biliar

Endoscopia gastroduodenal o colónica

Ablación tumoral con radiofrecuencia

Cateterismo arterial

Enema de bario

Ultrasonido endoscópico (debido a que la bilis fluye de la periferia hacia el hilio) con un patrón menos "enramado" o vascular. ${ }^{5}$ Si esto se complementa con ultrasonido, se puede apreciar cómo el gas biliar permanece estático. ${ }^{13}$ Cabe mencionar que la ausencia radiológica de neumobilia ha sido utilizada para predecir obstrucción de las prótesis biliares. Por otra parte, la imagen de gas portal, se define radiológicamente como la presencia de áreas de aspecto tubular con atenuación disminuida y localizadas hacia la periferia del parénquima hepático. Aunque el gas portal puede ser detectado en radiografías simples de abdomen, es mucho más fácil con la tomografía. Además, con el ultrasonido se logra apreciar cómo las burbujas de gas se desplazan en forma centrífuga hacia la periferia del hígado.

En los años 50 y 60, la detección de gas portal se realizaba exclusivamente con radiografías simples, las cuales requerían un volumen mayor de gas para ser visto y, por lo tanto, se asociaban a casos de mayor gravedad y alta mortalidad, llevando de la mano conductas agresivas como laparotomía de urgencia o procedimientos invasivos. Con el advenimiento de la tomografía, aumentó la sensibilidad diagnóstica y se ha visto su asociación a condiciones menos graves, lo cual permitió disminuir la mortalidad con tratamientos más conservadores. En la actualidad, se sugiere clasificar las causas de gas portal, según su riesgo de mortalidad en alto ( $>75 \%)$, moderado $(<30 \%)$ y bajo (prácticamente $0 \%$ ). Con base en estos niveles de riesgo, Nelson y colaboradores proponen un algoritmo de tratamiento que va desde el manejo agresivo hasta el conservador. $^{8}$

Aspectos terapéuticos relacionados a la presencia de neumobilia o gas venoso portal. La presencia de neumobilia no se considera, por sí sola, una indicación de laparotomía urgente o manejo agresivo. En términos generales, se puede ver que las causas de neumobilia se asocian a menor mortalidad inmediata y en este sentido se sugiere que se recurra en primera instancia a medidas terapéuticas no invasivas. ${ }^{1}$ En el caso de la presencia de gas venoso portal, al ser un hallazgo poco frecuente en el adulto, existen pocas series que estudian su abordaje terapéutico más apropiado. En una revisión retrospectiva de 11 pacientes se encontró que los casos se podían clasificar en dos grupos: gas portal asociado a neumatosis intestinal por tomografía, y otro donde no. Los primeros fueron los casos más graves (con isquemia intestinal y sepsis) requiriendo manejos más agresivos, mientras que los que no la tenían pudieron ser tratados de manera más conservadora. ${ }^{5}$ En otra serie se encontró que la presencia de gas portal en más de tres segmentos hepáticos tiene una sensibilidad del $100 \%$ para predecir un desenlace fatal, pero una especificidad del $50 \%$. El valor predictivo negativo (para muerte) de este hallazgo fue del $100 \%$ ( $p<0.005)$ y el valor predictivo positivo (para muerte) de la presencia de neumatosis intestinal fue también del 100\% ( $p<0.001$ ), por lo que concluyeron que la presencia de gas portal en dos o menos segmentos y la ausencia de neumatosis intestinal predicen un mejor desenlace. ${ }^{14}$ Sin embargo, al ser un hallazgo que no siempre se asocia a un estado clínico grave, desde 1986 se empezó a recomendar a los cirujanos a ser más conservadores en pacientes sin datos clínicos de sepsis o respuesta inflamatoria sistémica. ${ }^{15}$

\section{CONCLUSIÓN}

A pesar de existir criterios específicos para diferenciar entre neumobilia y gas portal, el diagnóstico preciso sigue siendo un reto que exige una cooperación estrecha entre radiólogos y clínicos. La determinación radiológica de gas en el sistema venoso o gas en los conductos biliares, debe ser un complemento para la evaluación detallada del es- 
tado general del paciente y así poder establecer el curso terapéutico más apropiado. La agresividad del tratamiento siempre debe ser guiada por la severidad propia de la patología del paciente y las condiciones que hagan sospechar sobre el origen de ésta. Un diagnóstico radiológico, tanto de neumobilia como de gas portal, que no corresponde con la situación clínica, no debe ser el único elemento para tomar una decisión terapéutica agresiva.

\section{REFERENCIAS}

1. Sherman SC, Tran H. Pneumobilia: benign or life-threatening. J Emerg Med. 2006; 30 (2): 147-153.

2. Abboud B, El Hachem J, Yazbeck T, Doumit C. Hepatic portal venous gas: physiopathology, etiology, prognosis and treatment. World J Gastroenterol. 2009; 15 (29): 3585-3590.

3. Franken JM, Veen EJ. Hepatic portal venous gas. J Gastrointestin Liver Dis. 2010; 19 (4): 360.

4. Wiesner W, Mortelé KJ, Glickman JN, Ji H, Ros PR. Pneumatosis intestinalis and portomesenteric venous gas in intestinal ischemia: correlation of CT findings with severity of ischemia and clinical outcome. AJR Am J Roentgenol. 2001; 177 (6): 1319-1323.

5. Peloponissios N, Halkic N, Pugnale M, Jornod P, Nordback P, Meyer A et al. Hepatic portal gas in adults: review of the literature and presentation of a consecutive series of 11 cases. Arch Surg. 2003; 138 (12): 1367-1370.

6. Kinoshita H, Shinozaki M, Tanimura H, Umemoto Y, Sakaguchi S, Takifuji $\mathrm{K}$ et al. Clinical features and management of hepatic portal venous gas: four case reports and cumulative review of the literature. Arch Surg. 2001; 136 (12): 1410-1414.
7. Liebman PR, Patten MT, Manny J, Benfield JR, Hechtman HB. Hepatic-portal venous gas in adults: etiology, pathophysiology and clinical significance. Ann Surg. 1978; 187 (3): 281-287.

8. Nelson AL, Millington TM, Sahani D, Chung RT, Bauer C, Hertl M et al. Hepatic portal venous gas: the ABCs of management. Arch Surg. 2009; 144 (6): 575-5781; discussion 581.

9. Griffiths DM, Gough MH. Gas in the hepatic portal veins. Br J Surg. 1986; 73 (3): 172-176.

10. Huurman VA, Visser LG, Steens SC, Terpstra OT, Schaapherder AF. Persistent portal venous gas. J Gastrointest Surg. 2006; 10 (5): 783-785.

11. Rajkovic Z, Papes D, Altarac S, Arslani N. Differential diagnosis and clinical relevance of pneumobilia or portal vein gas on abdominal X-ray. Acta Clin Croat. 2013; 52 (3): 369-373.

12. Lewandowski BJ, Withers C, Winsberg F. The air-filled left hepatic duct: the saber sign as an aid to the radiographic diagnosis of pneumobilia. Radiology. 1984; 153 (2): 329-332.

13. Pan HB, Huang JS, Yang TL, Liang HL. Hepatic portal venous gas in ultrasonogram--benign or noxious. Ultrasound Med Biol. 2007; 33 (8): 1179-1183.

14. Moussa M, Marzouk I, Abdelmoula K, Manamani A, Dali N, Farhat LC et al. Role of computed tomography in predicting prognosis of hepatic portal venous gas. Int J Surg Case Rep. 2017; 30: 177-182.

15. Ginesu GC, Barmina M, Cossu ML, Feo CF, Fancellu A, Addis F et al. Conservative approach to Hepatic Portal Venous Gas: A case report. Int J Surg Case Rep. 2017; 30: 183-185.

Conflicto de intereses: Los autores declaran no tener ningún conflicto de intereses.

Financiamiento: Los autores no recibieron patrocinio para llevar a cabo este artículo. 